\title{
The European Map of Axial Spondyloarthritis: Capturing the Patient Perspective-an Analysis of 2846 Patients Across 13 Countries
}

\author{
Marco Garrido-Cumbrera ${ }^{1,2,3}$ • Denis Poddubnyy ${ }^{4,5} \cdot$ Laure Gossec $^{6,7} \cdot$ David Gálvez-Ruiz $^{1,2} \cdot$ Christine Bundy $^{8}$. \\ Raj Mahapatra ${ }^{9}$ - Souzi Makri ${ }^{10}$ - Laura Christen ${ }^{11}$ - Carlos J. Delgado-Domínguez ${ }^{2}$ - Sergio Sanz-Gómez ${ }^{1,2}$.

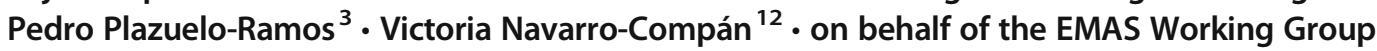

Published online: 12 March 2019

(C) The Author(s) 2019

\begin{abstract}
Purpose of Review Scientific research in axial spondyloarthritis (axSpA) has grown significantly. Nevertheless, the patient perspective remains insufficiently explored. Using a cross-sectional survey, the European Map of Axial Spondyloarthritis (EMAS) describes how patients living with self-reported axSpA experience their disease physically, psychologically, and socially.

Recent Findings 2846 patients participated: mean age $43.9 \pm 12.3$ years, $61.3 \%$ female, mean disease duration was $17.2 \pm$ 12.4 years, and $71.3 \%$ were HLA-B27 positive. Mean diagnostic delay was $7.4 \pm 8.4$ years. Mean BASDAI score was $5.5 \pm$ 2.0 and $75.7 \%$ reported moderate/severe spinal stiffness throughout the day. Daily life was substantially impaired: $74.1 \%$ reported difficulties finding a job due to the disease, and $61.5 \%$ reported psychological distress.

Summary EMAS results showed long diagnostic delay and substantial physical and psychological burden, indicating important unmet needs for patients. Furthermore, axSpA restricted patients' ability to participate in their daily routine and lead a productive work life. Understanding the patient's perspective can improve both health outcomes and shared decision-making between patient and rheumatologist.
\end{abstract}

Keywords Axial spondyloarthritis - Ankylosing spondylitis · Non-radiographic axial spondyloarthritis $\cdot$ Patient's perspective Burden of the disease $\cdot$ Shared decision-making $\cdot$ Europe

\section{Introduction}

Axial spondyloarthritis (axSpA) is a chronic inflammatory disease that encompasses radiographic (traditionally known as ankylosing spondylitis [AS]) and non-radiographic (nr-

This article is part of the Topical Collection on Spondyloarthritis

Marco Garrido-Cumbrera

mcumbrera@us.es

1 Universidad de Sevilla, Seville, Spain

2 Health \& Territory Research (HTR), Centro de Investigación, Tecnología e Innovación Manuel Losada Villasante (CITIUS), Universidad de Sevilla, C/ Dr Rafael Martínez Domínguez s/n, 41013 Seville, Spain

3 Spanish Federation of Spondyloarthritis Patient Associations (CEADE), Madrid, Spain

4 Charité-Universitätsmedizin Berlin, Berlin, Germany

5 German Rheumatism Research Centre, Berlin, Germany
axSpA) forms. This inflammatory disease can lead to chronic pain, structural damage, and disability [1]. In particular, the physical restrictions and worsening quality of life caused by the disease are closely related to the limitations that patients face in their professional, social, and family spheres [2,3], as
6 Sorbonne Université, Institut Pierre Louis d'Epidémiologie et de Santé Publique (iPLESP), Paris, France

7 Rheumatology Department, Pitié Salpêtrière hospital, AP-HP, Paris, France

8 Cardiff University, Cardiff, UK

9 Ankylosing Spondylitis International Federation (ASIF), London, UK

10 Cyprus League Against Rheumatism, Nicosia, Cyprus

11 Novartis Pharma AG, Patient Advocacy, Basel, Switzerland

12 IdiPaz, Hospital Universitario La Paz, Madrid, Spain 
well as the overall impact on psychological health, not only for patients living with axSpA but also for their families [4].

The insidious nature of the disease can be misleading, as periods of apparent disease inactivity can in fact be periods of great pain, stiffness, and fatigue for patients [5]. Such discrepancy, in many cases, leads patients to feel misunderstood or disregarded [6], and therefore less likely to share their experiences with others, including their physician. Consequently, patient disengagement results in patients being less involved in medical decisions as well as poor treatment adherence, poor health outcomes [3], worse course of the disease, and quality of life [7]. For these reasons, and as indicated in the update of the ASAS/EULAR recommendations for managing axSpA, considering the patient perspective in the management of their disease and ensuring patients are sufficiently prepared to participate in discussions are critical to treatment success and good adherence $[8 \cdot \bullet]$.

Nevertheless, recent research in the field of axSpA has largely focused on, and led to, an improved understanding of its clinical presentation and evolution of symptoms, specifically in disease activity and structural damage. Studies such as GErman SPondyloarthritis Inception Cohort (GESPIC) [9], the Outcome in AS International study (OASIS) conducted in France, Belgium and the Netherlands [10], the DEvenir des Spondylarthropathies Indifférenciées Récentes (DESIR) cohort in France [11], and the ESPeranza program for diagnosing early spondyloarthritis in Spain [12] have helped to strengthen scientific evidence and transform clinical practice. Although clinical studies often collect data on functional limitation, psychological distress, or working impact, they invariably do so using tools created with a clinical and not wholly patient perspective, thereby missing essential aspects relevant to patients and important to their optimal management.

European Map of Axial Spondyloarthritis (EMAS) aimed to generate evidence on patient-reported aspects of axSpA using a questionnaire developed in collaboration with patients, the Ankylosing Spondylitis International Federation (ASIF), clinical academic experts, describing how patients self-reporting as axSpA experience their disease from a physical, psychological, and social perspective and how they are managed within healthcare systems. We anticipate that the data gathered will help to highlight current unmet needs, including the need for early diagnosis, as well as inform personalized long-term disease management plans and treatment goals and to ultimately improve quality of life and optimize clinical outcomes for patients.

\section{Methods}

\section{Design and Survey Development}

EMAS was a cross-sectional survey of patients self-reporting as axSpA from Austria, Belgium, France, Germany, Italy, the
Netherlands, Norway, Russia, Slovenia, Sweden, Switzerland, the UK, and Spain. The survey was adapted from the Spanish Atlas of Axial Spondyloarthritis 2017 [13], a pilot survey held from January to March 2016 led by the Health \& Territory Research group of the University of Seville and including representatives from the Spanish Society of Rheumatology, the Spanish Federation of Spondyloarthritis Patient Associations (CEADE), the Max Weber Institute, and Novartis Farmacéutica Spain.

The EMAS questionnaire was originally developed in Spanish and subsequently translated into English followed by Dutch, French, German, Italian, Russian, Swedish, and Slovenian. Prior to the start of data collection, participating countries were asked to assess and modify questions for local relevance, with guidance to only make essential changes in order to maintain consistency on a pan-European level. Eight questions were removed from the original Atlas of Axial Spondyloarthritis in Spain 2017 survey template, as they were country-specific. The final patient questionnaire included 108 items related to 12 different areas: socio-demographic and anthropometric characteristics, disability assessment, work life, daily life, lifestyle habits, diagnostic journey, healthcare resource use, treatment, comorbidities (including extraarticular manifestations), psychological health, disease outcomes, and patient disease-related attitudes and treatment goals (see Table 1).

In addition, a range of supplementary indices were collected in the questionnaire to assess specific areas:

1. BASDAI (Bath Ankylosing Spondylitis Disease Activity Index) - a validated self-administered questionnaire assessing disease activity in patients with axSpA; relating to symptoms of fatigue; pain in the spinal column; inflammation/pain in joints other than the neck, back, and hips; areas of localized tenderness (also called enthesitis or inflammation of tendons and ligaments); and the level and duration of stiffness in the morning. Possible scores range from 0 (no activity) to 10 (maximum activity).

2. General Stiffness Index - this index, developed specifically for this study, assesses the degree of stiffness experienced by patients in the spinal column, distinguishing between the cervical, dorsal, and lumbar areas. Possible responses range from the least to the most affected column (1, without stiffness; 2 , mild stiffness; 3 , moderate stiffness; and 4, severe stiffness), total scores are obtained by adding together the responses in each of the areas of the spine without weighting resulting in a scale ranging from 3 to 12. This index showed an acceptable internal reliability $($ Cronbach alpha $=0.79)$.

3. Global Limitation Index - this index, developed specifically for this study, assesses the degree of limitation in 18 activities of daily life (dressing, bathing, showering, tying 
Table 1 Areas, variables, and measurements/categories included within the EMAS patient questionnaire

\begin{tabular}{|c|c|c|}
\hline Area & Variable & Measurement/categories \\
\hline \multirow{11}{*}{$\begin{array}{l}\text { Socio-demographic and } \\
\text { anthropometric characteristics }\end{array}$} & Country of residency & Name of the country \\
\hline & Age & Years \\
\hline & Gender & Female, male \\
\hline & Marital status & Single, married, separate/divorced, widowed \\
\hline & $\begin{array}{l}\text { Number of children, number of family } \\
\text { members }\end{array}$ & Numerical \\
\hline & Relationship status & Yes, no \\
\hline & Educational level & No schooling, primary, high school, university \\
\hline & Household income level & $\begin{array}{l}\text { Euros per month; household income level per capita, calculated dividing } \\
\text { this value by number of household members }\end{array}$ \\
\hline & Membership to patients association & Yes, no \\
\hline & Weight and height & $\mathrm{Kg}$ and $\mathrm{cm}$ \\
\hline & & Body mass index, calculated from these two indicators \\
\hline \multirow[t]{3}{*}{ Disability assessment } & Assessment of disability & Yes, no \\
\hline & Degree of disability & Yes, no \\
\hline & Social security benefits & Yes (type of security benefit), no \\
\hline \multirow[t]{6}{*}{ Work life } & Employment status & List of 8 professional status \\
\hline & Main occupation & List of 11 occupations \\
\hline & Hours per week in main occupation & Numerical \\
\hline & Work-related issues & $\begin{array}{l}\text { List of } 7 \text { work-related issues: asked for days off (number of days), took } \\
\text { sick leave (number of days), reduced working hours (number of hours), } \\
\text { missed work for doctor appointments, difficulty fulfilling working } \\
\text { hours, changed work shift, suffering of professional life (yes, no) }\end{array}$ \\
\hline & Employment status due to axSpA & Yes, no \\
\hline & Job loss due to axSpA & Yes, no \\
\hline \multirow[t]{5}{*}{ Daily life } & Functional limitation in daily activities & Degree of functional limitation in 18 daily life activities \\
\hline & Help needed in daily activities & Frequency of help needed in 18 daily life activities \\
\hline & Impact on social relationships & $\begin{array}{l}\text { List of } 5 \text { social relationship (much better than before, better than before, } \\
\text { same as before, worse than before, much worse than before) }\end{array}$ \\
\hline & Frequency of leisure/cultural activites & $\begin{array}{l}\text { List of } 5 \text { leisure/cultural activities (much more than before, more than } \\
\text { before, same as before, less than before, much less than before) }\end{array}$ \\
\hline & Adaptations since disese onset & $\begin{array}{l}\text { List of } 5 \text { adaptations: adapting your workplace, moved to another job, } \\
\text { adapting your home, adapting your car, customized shoes (yes, no) }\end{array}$ \\
\hline \multirow[t]{5}{*}{ Lifestyle habits } & Physical exercise & List of 15 physical activities (yes, no and number of hours) \\
\hline & Visited health spa & Yes, no \\
\hline & Money spent on rehabilitation & Amount in euros \\
\hline & Smoking & $\begin{array}{l}\text { Non smoker, sporadically/socially, fewer than } 10 \text { cigarettes per week, } \\
10-20 \text { cigarettes per week, } 21-60 \text { cigarettes per week, over } 60 \\
\text { cigarettes per week }\end{array}$ \\
\hline & Alcohol & $\begin{array}{l}\text { Never, ocassionally, } 1-3 \text { times per month, } 1-2 \text { times per week, } 3-5 \text { times } \\
\text { per week, every day }\end{array}$ \\
\hline \multirow[t]{7}{*}{ Diagnostic journey } & Age of onset of symptoms & Numerical \\
\hline & Age at diagnosis & Numerical \\
\hline & HCP who made the diagnosis & List of 4 HCPs \\
\hline & HCP seen before diagnosis & List of 6 HCPs (yes, no, other) \\
\hline & First tests for diagnosis & $\begin{array}{l}\text { List of } 6 \text { medical tests: MRI scan, X-rays, genetic analysis, ultrasound } \\
\text { scan, radionuclide scintigraphy, CT scan (yes, } \\
\text { no, other) }\end{array}$ \\
\hline & Result of HLA-B27 & Positive, negative, do not know \\
\hline & Familiars with axSpA & Kinship and number \\
\hline \multirow[t]{2}{*}{ Healthcare resource use } & Main health insurance & Public, private, out-of-pocket, other \\
\hline & & List of $10 \mathrm{HCPs}$ (numerical) \\
\hline
\end{tabular}


Table 1 (continued)

\begin{tabular}{|c|c|c|}
\hline Area & Variable & Measurement/categories \\
\hline & $\begin{array}{l}\text { Number of visits to health professionals } \\
\text { in the past } 12 \text { months }\end{array}$ & \\
\hline & $\begin{array}{l}\text { Number of medical tests for follow-up } \\
\text { in the past } 12 \text { months }\end{array}$ & List of 7 medical tests (numerical) \\
\hline & $\begin{array}{l}\text { Number of inpatient admissions in the } \\
\text { past } 12 \text { months }\end{array}$ & Numerical \\
\hline & $\begin{array}{l}\text { Number of uses of emergency services } \\
\text { in the past } 12 \text { months }\end{array}$ & $\begin{array}{l}\text { List of } 4 \text { emergency services: hospital, healthcare centre/outpatient clinic, } \\
\text { home emergency, ambulance (numerical) }\end{array}$ \\
\hline \multirow[t]{6}{*}{ Treatment } & Pharmacological & List of 3 treatments: biological therapy, NSAIDs, and DMARDs (Yes, no) \\
\hline & & Impact on 9 areas (score from 0 to 10 ) \\
\hline & Visited health spa & Yes, no \\
\hline & Money spent on rehabilitation & Amount in euros \\
\hline & Alternative treatments & Acupuncture, homeopathy, none, other. \\
\hline & Discussion of treatment goals with $\mathrm{HCP}$ & Yes, no \\
\hline \multirow[t]{2}{*}{$\begin{array}{l}\text { Comorbidities and extra-articular } \\
\text { manifestations }\end{array}$} & Comorbidities associated to axSpA & $\begin{array}{l}\text { List of } 27 \text { comorbidities: psoriatic arthritis, uveitis, episcleritis, gout, } \\
\text { fibromyalgia, spinal or other fractures, liver disease, genital lesions, } \\
\text { hypertension, hypercholesterolemia, diabetes, kidney failure, heart } \\
\text { failure, cataracts, glaucoma, irregular heart beat, pacemaker fitted, } \\
\text { coronary artery disease, atherosclerosis, any severe infection requiring } \\
\text { inpatient hospital admission, any severe infections requiring } \\
\text { antibiotics, sleep disorders, depression, anxiety, obesity/overweight } \\
\text { (Yes, no) }\end{array}$ \\
\hline & Extra-articular manifestations & $\begin{array}{l}\text { Uveitis and inflammatory bowel disease (ulcerative colitis, Chron's } \\
\text { disease) (Yes, no) }\end{array}$ \\
\hline \multirow[t]{3}{*}{ Psychological health } & Psychological distress & 12-item General Health Questionnaire (GHQ-12) (0-12) \\
\hline & $\begin{array}{l}\text { Presence of anxiey, depression, or sleep } \\
\text { disorders }\end{array}$ & Yes, no \\
\hline & $\begin{array}{l}\text { Visits to psychologists/psychiatrists in } \\
\text { the past } 12 \text { months }\end{array}$ & Numerical \\
\hline \multirow[t]{5}{*}{ Disease outcomes } & Disease activity & Bath Ankylosing Spondylitis Disease Activity Index (BASDAI) (0-10) \\
\hline & Body areas with inflammation & Inflammation in body areas (yes, no) \\
\hline & Spinal stiffness & Patient-reported stiffness or ankylosis in the spine (yes/no) \\
\hline & & Degree of restriction in cervical, dorsal and lumbar areas $(1-4)$ \\
\hline & & Global Stiffness Index (3-12) \\
\hline \multirow{3}{*}{$\begin{array}{l}\text { Patient disease-related attitudes } \\
\text { and treatment goals }\end{array}$} & Fears related to axSpA & One open-ended question \\
\hline & Hopes related to axSpA & One open-ended question \\
\hline & Treatment goals related to axSpA & One open-ended question \\
\hline
\end{tabular}

shoe laces, moving about the house, climbing stairs, getting out of bed, using the bathroom, shopping, preparing meals, eating, household cleaning, walking down the street, using public transportation, driving, going to the doctor, doing physical exercise, having sex). Each of these 18 activities was assigned as 0 for no limitation, 1 low limitation, 2 medium limitation and 3 high limitations, resulting in values between 0 and 54. A total score from 0 and 18 was considered low limitation, between 18 and 36 medium limitation, and between 36 and 54 high limitation. Cronbach alpha of 0.97 demonstrated excellent internal reliability.

4. GHQ-12 (General Health Questionnaire-12) —-this questionnaire evaluates psychological distress using 12 questions. For the present study, these were transformed into a dichotomous score (0-0-1-1), called the GHQ score, to eliminate any bias resulting from the tendency of the respondents to choose answers 1 and 4 or 2 and 3. The cutoff point of 3 implied those with a score of 3 or more may be experiencing psychological distress [14].

\section{Sample Selection and Recruitment}

The sample selection inclusion criteria were as follows:

- $\quad$ aged $\geq 18$ years,

- residents of the specified European country, 
- a self-reported diagnosis of axSpA, including Ankylosing Spondylitis or non-radiographic axSpA,

- visit to a healthcare professional for $\operatorname{axSpA}$ in the 12 months prior to participation.

Participants were recruited between July 2017 and March 2018 by GfK through their existing database of respondents. In Austria, Norway, Slovenia, Sweden, the Netherlands, Italy, and Russia, Patient Advocacy Groups (PAGs) also supported recruitment by distributing the survey to their members. The questionnaire was completed via an online platform for survey data collection. In addition, the database from the Atlas of Axial Spondyloarthritis in Spain 2017 [15•] was retrospectively added to the EMAS database.

\section{The EMAS Working Group}

The EMAS project is a collaboration led by the Health \& Territory Research group of the University of Seville, ASIF, and a steering committee composed of patient representatives and internationally recognized rheumatologists, psychologists, and researchers specialized in axSpA.

\section{Results}

\section{Participation Rate and Socio-demographics}

A total of 2846 people with self-reported axSpA participated in the EMAS survey. Figure 1 presents the distribution of participants by country, with the largest sample sizes found in Spain, France, Norway, and Russia in that order; EMAS patient socio-demographic, anthropometric characteristics, and lifestyle habits are summarized in Table 2. Overall, three out of five participants were female $(61.4 \%)$ with a mean (SD) age of 44 (12) years. The majority of participants were either married or in a relationship and were at least high school educated.

\section{Disease-Specific Characteristics}

Disease characteristics are depicted in Table 3. The majority of participants reported a diagnosis of AS (79.2\%), while the remainder reported being diagnosed with nr-axSpA $(8.5 \%)$ or just $\operatorname{axSpA}$ without specifying the subtype (12.3\%). The average age of symptom onset was 26.2 (11.1) years, the mean disease duration was 17.2 (12.2) years, and the mean diagnostic delay reported was $7.4(8.4)$ years. Seventy-one percent of those who reported their HLA-B27 status stated that they were HLA-B27 positive. Around $20 \%$ of participants reported a diagnosis of an extra-articular manifestation, comprising uveitis or inflammatory bowel disease.
The mean BASDAI score was 5.5 (2.0), with the majority of participants reporting at least moderate spinal stiffness and $50.1 \%$ reporting medium to high functional limitation during disease flares.

\section{Working Life, Psychological Health, and Disease-Related Attitudes}

Results of the impact of axSpA on working life, psychological health, and patient disease-related perceptions are summarized in Table 4. Nearly half of the participants reported that their disease influenced their job choice and $74.1 \%$ reported having difficulties finding a job due to the disease. Additionally, more than half of the participants reported psychological distress $(61.5 \%)$, with one out of three reporting anxiety and/or depression.

Participants commonly reported fear of disease progression, fear of suffering pain, or loss of mobility. Participants' hopes were mainly to halt disease progression, to eliminate pain, and to receive effective treatment. However, one third of the participants surveyed reported that they had not talked to their clinician about their personal treatment goals.

\section{Discussion}

In this very large European sample, the observed data indicate important unmet needs in axSpA, including long diagnostic delay, deterioration of quality of life, and high burden of disease for patients. First, there is an ongoing and critical need for early and accurate diagnosis. The EMAS diagnostic delay was calculated at over 7 years and confirmed the results of a metaanalysis conducted by Jovaní et al., which found the diagnostic delay to be 8.8 years for females and 6.5 years for males [16]. Furthermore, EMAS results showed that patients on average visited two healthcare professionals, mainly general practitioners (GPs), followed by orthopedic specialists, physiotherapists, and osteopaths (excluding rheumatologists), prior to receiving a diagnosis. It is therefore necessary to improve disease education among healthcare professionals, specifically those responsible for referring patients to a rheumatologist (e.g., primary care physicians, physiotherapists, orthopedic surgeons), as well as optimizing collaboration between them in order to shorten the patient journey to diagnosis, and ultimately effective treatment.

EMAS results also showed a high burden of disease for patients. The majority of participants reported moderate to severe limitation during disease flares, which was especially evident while performing daily activities including physical exercise, cleaning, getting out of bed, or getting dressed. Participants also reported difficulties finding a job due to their $\operatorname{axSpA}(74.1 \%)$, that the disease influenced their job choice (45.7\%), and that they required workplace adaptation (43.9\%). 


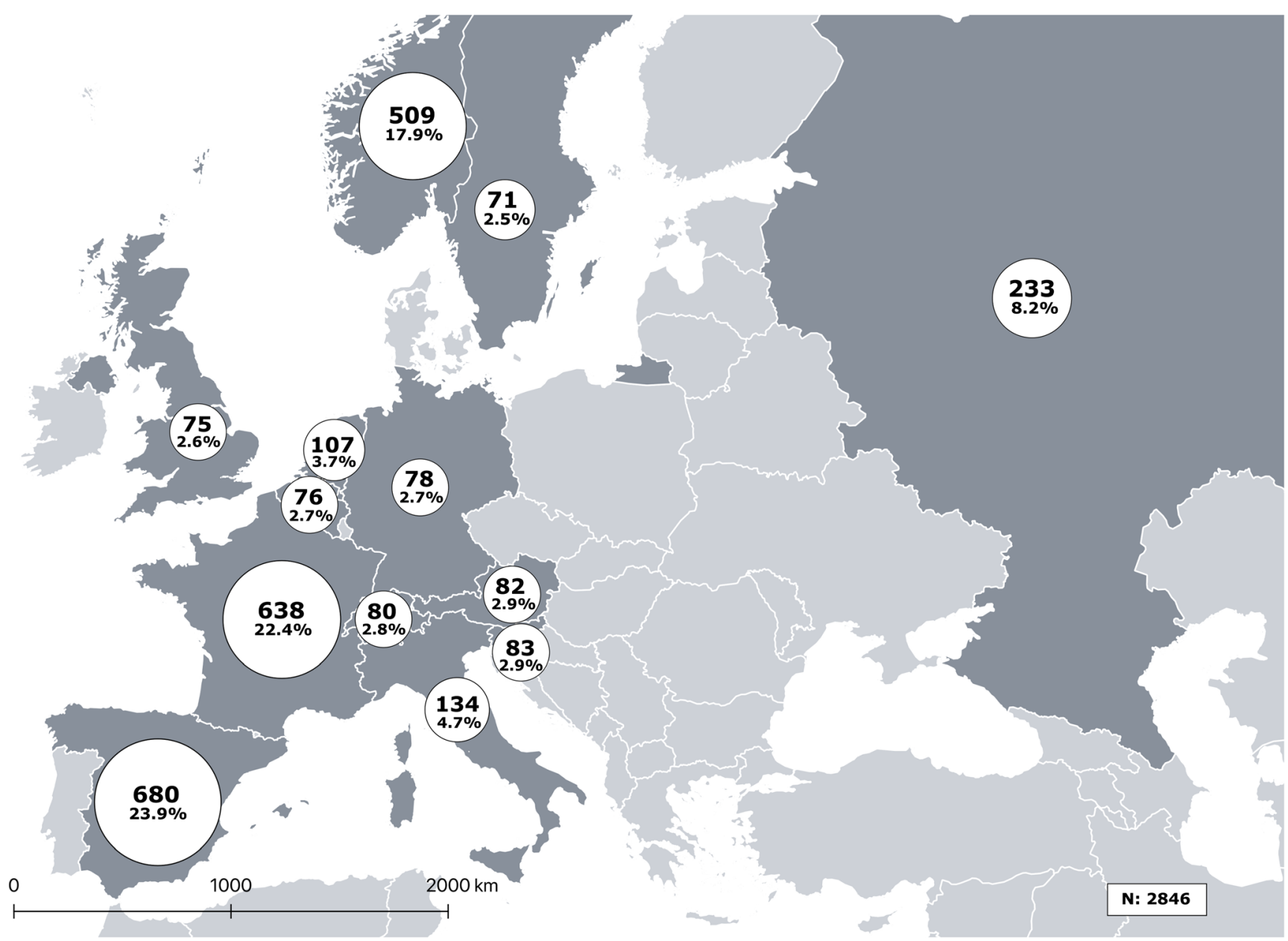

Fig. 1 Distribution of EMAS survey participants by European country

As in previous studies [17], the EMAS sample showed a high prevalence of mental health difficulties. $61.5 \%$ of the sample was at risk for psychological distress, with 33.8\% and $38.6 \%$ respectively reporting depression and/or anxiety. This contrasts with the WHO prevalence rates, in which anxiety within European participating countries is reported to be between $3.1 \%$ (Russia) and 7.4\% (Norway) and depression between $4.5 \%$ (UK) and 5.5\% (Russia).

Additionally, though previous studies have explored axSpA patient personal hopes and fears related to the disease using quantitative questionnaires [18], EMAS adopted a qualitative approach to understand these factors. When asked to state their disease-related hopes and fears, EMAS participants most frequently reported fear of and hope of stopping disease progression and pain. This is understandable as patients with axSpA suffer from a high degree of anxiety and uncertainty due to the unpredictability of disease flares [5].

These axSpA-related hopes and fears may consequently influence several factors including the patientphysician relationship or treatment adherence [19]; it is critical for patients to share these with their physician. Equally important to the patient-physician dialog is the discussion of the patient's personal treatment goals. One in three EMAS participants had not discussed their personal treatment goals with their physician. Ultimately, both healthcare professionals and patients should be encouraged to engage in a proactive discussion regarding expectations and goals for axSpA treatment to enable effective shared decision-making and the design of individualized treatment strategies that provide optimal management of the disease [20].

EMAS is the largest survey carried out to date for people with axSpA, across 2846 respondents from 13 European countries. The EMAS focus was on understanding the patient perspective through a holistic approach and utilizing a questionnaire designed for patients, by patients. As such, EMAS collected not only clinical characteristics of the disease but also the impact this had on patient's psychological health, daily activities, and working and social life as well as how the disease relates to their hopes and fears, all of which are considered relevant and important aspects to patients with axSpA.

We acknowledge that EMAS has some limitations. First, the survey relied on self-reported data, and did not attempt to 
Table 2 Socio-demographic, anthropometric characteristics, and lifestyle habits

\begin{tabular}{ll}
\hline Variable, $n$ patients with data available & Mean \pm SD $/ n(\%)$ \\
\hline Age (years) $n=2846$ & $43.9 \pm 12.3$ \\
Gender (female), $n=2846$ & $1746(61.4)$ \\
Marital status, $n=2846$ & \\
Single & $601(21.1)$ \\
Married & $1933(67.9)$ \\
Separated/divorced & $273(9.6)$ \\
Widowed & $39(1.4)$ \\
Educational level, $n=2846$ & \\
No schooling completed & $32(1.1)$ \\
Primary school & $263(9.2)$ \\
High school & $1181(41.5)$ \\
University & $1370(48.1)$ \\
Monthly income (euros) per household & $1122.6 \pm 902.7$ \\
member, $n=2289$ & \\
BMI, $n=2846$ & $108(3.8)$ \\
Underweight $(<18.5)$ & $1252(44.0)$ \\
Normal weight $(18.5-24.9)$ & $953(33.5)$ \\
Overweight (25-29.9) & $533(18.7)$ \\
Obesity (>30) & $1851(67.3)$ \\
Smoking, $n=2751$ & $380(13.8)$ \\
Non smoker & $520(18.9)$ \\
Less than 10 cigarettes/day & \\
More than 10 cigarettes & $196(7.1)$ \\
Alcohol consumption, $n=2751$ & \\
Never or occasionally & \\
$1-2$ times per week & \\
More than twice per week & \\
Member of a patient support group, $n=2846.9)$ \\
\hline
\end{tabular}

confirm participant diagnosis nor to support participant responses with clinician reported assessments. As such, clinical data such as the BASDAI or GHQ-12 scores may also suffer from response bias. Nevertheless, the sample characteristics were consistent with previous cohorts including patients with confirmed axSpA [9-12], and as the aim of the survey was to better understand the patient perspective, direct feedback was preferred.

Secondly, we used some non-validated scales or indices for assessing certain factors, such as functional limitations in daily activities and spinal stiffness. The reason for utilizing such scales or composite indices originated during the preliminary phase of the survey development, when patients expressed their concern about not being able to report all aspects of their disease if other scales or indices were to be employed. In any case, a good Cronbach alpha value was obtained for the indices employed in EMAS, which support the reliability of these instruments in this sample. Lastly, the differences in sample sizes between countries, resulting from the two recruitment
Table 3 Disease-specific characteristics

\begin{tabular}{ll}
\hline Variable, $n$ patients with data available & Mean $\pm \mathrm{SD} / n(\%)$ \\
\hline Type of condition, $n=2846$ & \\
Ankylosing spondylitis & $2254(79.2)$ \\
Non-radiographical axial spondyloarthritis & $304(8.5)$ \\
Unspecified axial spondyloarthritis & $288(12.3)$ \\
Age at onset of first symptoms, years, $n=2721$ & $26.2 \pm 11.1$ \\
Age at diagnosis, years, $n=2722$ & $33.7 \pm 11.5$ \\
Diagnostic delay, years $n=2652$ & $7.4 \pm 8.4$ \\
Disease duration, years $n=2716$ & $17.2 \pm 12.4$ \\
Extra-articular manifestations, $n=2096$ & \\
Uveitis & $469(22.4)$ \\
Inflammatory bowel disease & $294(14.0)$ \\
HLA-B27 (positive), $n=1799$ & $1283(71.3 \%)$ \\
BASDAI (0-10) $n=2584$ & $5.5 \pm 2.0$ \\
Spinal Stiffness Index (3-12), $n=2660$ & $7.7 \pm 2.5$ \\
Maximum degree of stiffness, $n=2707$ & \\
No stiffness & $187(6.9 \%)$ \\
Mild & $471(17.4 \%)$ \\
Moderate & $934(34.5 \%)$ \\
Severe & $1115(41.2 \%)$ \\
Global Limitation Index $(0-54), n=2771$ & \\
Overall limitation & $20.4 \pm 16.3$ \\
Low (0-17) & $1383(49.9 \%)$ \\
Medium (18-35) & $587(28.9 \%)$ \\
High (36-54) & \\
\hline & \\
&
\end{tabular}

methods employed (GfK online panel and patient groups), naturally skew the aggregate data towards the experiences of patients in countries with greater sample weight.

Despite these limitations, EMAS adopts a multidisciplinary approach, including the medical and patient community within the research team and aiming to understand the patient experience from a holistic perspective. Results from EMAS were presented at the 13th General Council Meeting of ASIF 2018 held in Guangzhou (China) during which the implications of the findings were discussed with patient and rheumatologist leaders from around the world. They were also disseminated at professional congresses, including EULAR 2018 held in Amsterdam, International Congress on Spondyloarthritis (ICS) 2018 in Ghent, 2018 French Rheumatology Congress (SFR) in Paris, and ACR 2018 in Chicago, in order to enhance interest in better understanding of the patient perspective within the scientific community. Continuing its momentum, the EMAS survey and vision are currently being expanded globally as the International Map of Axial Spondyloarthritis (IMAS), including Canada, the USA, Mexico, Costa Rica, Colombia, Argentina, South Korea, Taiwan, and Turkey. By broadening the scope of the 
Table 4 Working life, psychological health, and disease-related perceptions

\begin{tabular}{ll}
\hline Variable, $n$ patients with data available & Mean $\pm \mathrm{SD} / n$ \\
& $(\%)$ \\
\hline
\end{tabular}

Employment status of labor force, $n=1653$

Employed

1457 (87.7)

Unemployed

Employment status of economically inactive, $n=1042$

Temporary sick leave

$304(29.2)$

Permanent sick leave

$292(28.0)$

Retired

$230(22.1)$

Early retirement

$43(4.1)$

Homemaker

114 (10.9)

Student

$59(5.7)$

Required a workplace adaptation due to

$1163(43.9 \%)$ axSpA, $n=2651$

AxSpA influenced job choice, $n=2527$

$1156(45.7 \%)$

Difficulties finding a job due to $\operatorname{axSpA}, n=2071$

$1534(74.1 \%)$

Psychological and sleep comorbidities, $n=2096$

Sleep disorder

$1058(50.5)$

Anxiety

809 (38.6)

Depression

$710(33.9)$

$4.9 \pm 4.1$

GHQ score, $(0-12) n=2640$

$1624(61.5 \%)$

At risk for psychological distress (GHQ $\geq 3$ ), $n=2640$

801 (32.9)

Disease progression

$743(30.5)$

Suffering pain

$730(30.0)$

Loss of mobility

$791(32.5)$

Stop disease progression

$748(30.7)$

Eliminate pain

$567(23.3)$

Effective treatments

696 (28.6)

469 (19.3)

$202(8.3)$

1663 (66.6)

holistically managed, including access to therapies such as exercise programs, psychological, and physiotherapeutic care. EMAS also reaffirms the need to incorporate the patient's perspective into clinical practice, as it facilitates shared decision-making between patients and physicians, which improves disease management, increases patient participation in their care, ensures greater therapeutic adherence, and generates better physical and psychological health outcomes.

\section{Compliance with Ethical Standards}

Conflict of Interest EMAS was funded by Novartis Pharma AG. All authors have received honoraria for participating in EMAS from Novartis Pharma AG.

Dr. Denis Poddubnyy has received unrelated research grants from Abbvie, MSD, Novartis, and Pfizer, and unrelated honoraria from Abbvie, BMS, Celgene, Janssen, Lilly, MSD, Novartis, Pfizer, Roche, and UCB.

Dr. Laure Gossec has received unrelated research grants from BMS, Lilly, and Pfizer and unrelated honoraria from Abbvie, BMS, Celgene, Janssen, Lilly, Novartis, MSD, Pfizer, and UCB.

Dr. Christine Bundy has received unrelated honoraria from Abbvie, Celgene, Janssen, Lilly, Novartis, and Pfizer.

Dr. Victoria Navarro-Compán has received unrelated honoraria or research grants from Abbvie, BMS, Lilly, MSD, Novartis, Pfizer, Roche, and UCB.

Human and Animal Rights and Informed Consent This article does not contain any studies with animal subjects. All participants were asked to provide explicit opt-in consent prior to participating in the EMAS survey. Participant data was anonymized.

Open Access This article is distributed under the terms of the Creative Commons Attribution 4.0 International License (http:// creativecommons.org/licenses/by/4.0/), which permits unrestricted use, distribution, and reproduction in any medium, provided you give appropriate credit to the original author(s) and the source, provide a link to the Creative Commons license, and indicate if changes were made.

Publisher's Note Springer Nature remains neutral with regard to jurisdictional claims in published maps and institutional affiliations.

\section{References}

survey outside of Europe, the IMAS project will seek to describe the burden of disease from the perspective of patients around the world.

\section{Conclusion}

By highlighting the important limitations and disease burden that participants face in their daily life, EMAS emphasizes the need to take urgent measures to reduce the burden of disease associated with axSpA by reducing diagnostic delay and ensuring that patients are optimally and

Papers of particular interest, published recently, have been highlighted as:

- Of importance

•• Of major importance

1. Sieper J, Poddubnyy D. Axial spondyloarthritis. Lancet. 2017;390: 73-84.

2. Song $\mathrm{Y}$, Wang $\mathrm{C}$, Chen $\mathrm{H}$. Functional limitation and associated factors in outpatients with ankylosing spondylitis in Southwest China. Clin Rheumatol. 2017;36:871-7.

3. Shen CC, Hu LY, Yang AC, Kuo BI-T, Chiang YY, Tsai SJ. Risk of psychiatric disorders following ankylosing spondylitis: a 
nationwide population-based retrospective cohort study. J Rheumatol. 2016;43:625-31.

4. Rohde G, Berg KH, Prøven A, Haugeberg G. The relationship between demographic- and disease-related variables and healthrelated quality of life in patients with axial spondyloarthritis. BMC Musculoskelet Disord. 2017;18:328.

5. Khan MA. Ankylosing spondylitis: introductory comments on its diagnosis and treatment. Ann Rheum Dis. 2002;61:iii3-7.

6. Hamilton-West KE, Quine L. Living with ankylosing spondylitis: the patient's perspective. J Health Psychol. 2009;14:820-30.

7. Wang CTM, Fong W, Kwan YH, Phang JK, Lui NL, Leung YY, et al. A cross-sectional study on factors associated with patientphysician discordance in global assessment of patients with axial spondyloarthritis: an Asian perspective. Int J Rheum Dis. 2018;21: $1436-42$

8.• van der Heijde D, Ramiro S, Landewé R, Baraliakos X, Van den Bosch F, Sepriano A, et al. 2016 update of the ASAS-EULAR management recommendations for axial spondyloarthritis. Ann Rheum Dis. 2017;76:978-91. This document presents the 2016 ASAS-EULAR recommendations for the management of patients with axSpA.

9. Poddubnyy D, Haibel H, Listing J, Märker-Hermann E, Zeidler H, Braun J, et al. Baseline radiographic damage, elevated acute-phase reactant levels, and cigarette smoking status predict spinal radiographic progression in early axial spondylarthritis. Arthritis Rheum. 2012;64:1388-98

10. Ramiro S, Van Der Heijde D, Van Tubergen A, Stolwijk C, Dougados M, Van Den Bosch F, et al. Higher disease activity leads to more structural damage in the spine in ankylosing spondylitis: 12-year longitudinal data from the OASIS cohort. Ann Rheum Dis. 2014;73:1455-61.

11. Dougados M, d'Agostino MA, Benessiano J, Berenbaum F, Breban M, Claudepierre P, et al. The DESIR cohort: a 10-year follow-up of early inflammatory back pain in France: study design and baseline characteristics of the 708 recruited patients. Jt Bone Spine. 2011;78:598-603.

12. Collantes E, Zarco P, Muñoz E, Juanola X, Mulero J, FernándezSueiro JL, et al. Disease pattern of spondyloarthropathies in Spain: description of the first national registry (REGISPONSER) extended report. Rheumatology. 2007;46:1309-15.

13. Garrido-Cumbrera M, Navarro-Compán V, Zarco P, CollantesEstévez E, Gálvez-Ruiz D, Braçe O, et al. Atlas of axial spondyloarthritis in Spain 2017: study design and population. Reumatol Clin. 2018. https://doi.org/10.1016/j.reuma.2018.08.003.

14. Cano A, Sprafkin RP, Scaturo DJ, Lantinga LJ, Fiese BH, Brand F. Mental health screening in primary care: a comparison of 3 brief measures of psychological distress. Prim Care Companion J Clin Psychiatry. 2001;3:206-10.

15. Garrido Cumbrera M, Gálvez Ruiz D, Chacón García J, Braçe O, Villoro Valdés R, Merino Ventosa M, et al. Atlas of Axial Spondyloarthritis in Spain 2017. Profile of the disease. Madrid: Instituto Max Weber; 2017. This is the Atlas of axSpA report, which methodology was used as a pilot for the EMAS project.

16. Jovaní V, Blasco-Blasco M, Ruiz-Cantero MT, Pascual E. Understanding how the diagnostic delay of spondyloarthritis differs between women and men: a systematic review and metaanalysis. J Rheumatol. 2017;44:174-83.

17. Baysal Ö, Durmuș B, Ersoy Y, Altay Z, Șenel K, Nas K, et al. Relationship between psychological status and disease activity and quality of life in ankylosing spondylitis. Rheumatol Int. 2011;31:795-800.

18. Gossec L, Chauvin P, Hudry C, Mathoret-Philibert F, Poussière M, de Chalus T, et al. The most frequent fears and beliefs of 226 patients with rheumatoid arthritis or spondyloarthritis, using a novel questionnaire. Ann Rheum Dis. 2015;74:408.

19. Gossec L, Chauvin P, Saraux A, Hudry C, Cukierman G, de Chalus $\mathrm{T}$, et al. Development and psychometric validation of a patientreported outcome measure to assess fears in rheumatoid arthritis and axial spondyloarthritis: the Fear Assessment in Inflammatory Rheumatic diseases (FAIR) questionnaire. Ann Rheum Dis. 2018;77:258-63.

20. Smolen JS, Schöls M, Braun J, Dougados M, FitzGerald O, Gladman DD, et al. Treating axial spondyloarthritis and peripheral spondyloarthritis, especially psoriatic arthritis, to target: 2017 update of recommendations by an international task force. Ann Rheum Dis. 2018;77:3-17. 